\title{
ELÉMENTS DE LA PHYSIQUE DES PLASMAS ET MESURE ÉLECTROMAGNÉTIQUE
}

\section{ELÉMENTS DE PHYSIQUE DES PLASMAS}

Dans de nombreux chapitres de ce livre, nous avons donné des exemples tirés d'expériences avec le radar à diffusion incohérente EISCAT. Le principe de la diffusion incohérente sort du cadre de l'aéronomie (nous proposons une bibliographie succincte à ce sujet). Cependant, nous allons aborder les principes fondamentaux de la physique des plasma, qui permettent d'en comprendre le fonctionnement d'une manière approchée. Dans un premier temps, cela va nous amener à définir la longueur de Debye, puis la fréquence de plasma, ou onde électronique. Le pendant ionique sera alors calculé. Nous aborderons alors succinctement le principe de diverses méthode de sondage ionosphérique, dont la diffusion incohérente

\section{LONGUEUR DE DEBYE}

On considère un plasma composé d'une espèce ionique monochargée et d'électrons, et on suppose les ions immobiles (vrai en première approximation du fait du rapport des masses égal à 1832). A l'équilibre, la neutralité électrique s'exprime par : $\bar{n}_{i}=\bar{n}_{e}$, où le symbole '-' exprime une grandeur moyenne. Les électrons sont attirés par les ions, mais repoussés par les électrons. La conséquence de cela est une concentration d'électrons autour des ions qui va avoir pour effet, au moins à grande distance, de neutraliser le champ créé par l'ion positif ; c'est l'effet d'écran de Debye, qui écrante le potentiel de Coulomb à grande distance.

Supposons qu'à une distance $r$ de l'ion, la densité électronique soit $\tilde{n}_{e}(r)$, différente de la densité électronique moyenne $\bar{n}_{e}$. En supposant les électrons en équilibre thermodynamique à la température $T_{e}$, ces derniers se répartissent suivant le facteur de Boltzmann, soit :

$\tilde{n}_{e}(r)=\bar{n}_{e} \mathrm{e}^{-\frac{E(r)}{k_{b} T_{e}}}$

AIII-1

où $E(r)$ est l'énergie de l'électron à la distance $r$ de l'ion. Etant donné la faible masse de l'électron, cette énergie est essentiellement potentielle et est due à l'attraction coulombienne 
ion-électron, qui crée un potentiel $\phi(r)$ à la distance $r$ de l'ion. En conséquence, la concentration électronique s'exprime de la manière suivante :

$\tilde{n}_{e}(r)=\bar{n}_{e} \mathrm{e}^{\frac{e \phi(r)}{k_{b} T_{e}}}$

où $e$ est la charge élémentaire.

Pour une petite perturbation par rapport à l'équilibre, l'énergie potentielle d'interaction est faible devant l'énergie thermique $\left(\left|\frac{e \phi(r)}{k_{b} T_{e}}\right| \ll<1\right)$, de telle sorte que l'équation $A I I I-2$ se simplifie et devient :

$\tilde{n}_{e}(r)=\bar{n}_{e}\left(1+\frac{e \phi(r)}{k_{b} T_{e}}\right)$

D'autre part, ce potentiel $\phi$ doit localement vérifier l'équation de Poisson :

$\nabla^{2} \phi=-\frac{\rho}{\varepsilon_{\mathrm{o}}}=-\frac{\bar{n}_{i} e-\tilde{n}_{e}(r) e}{\varepsilon_{\mathrm{o}}}$

soit encore :

$\nabla^{2} \phi=e \frac{\tilde{n}_{e}(r)-\bar{n}_{e}}{\varepsilon_{\mathrm{o}}}=\frac{\bar{n}_{\mathrm{e}} e^{2}}{\varepsilon_{\mathrm{o}} k_{b} T_{e}} \phi(r)$

on introduit alors :

$\lambda_{D}=\sqrt{\frac{\varepsilon_{\mathrm{o}} k_{b} T_{e}}{\bar{n}_{e} e^{2}}}$

AIII-6

que l'on appelle la longueur de Debye. Cette longueur peut être approchée par :

$\lambda_{D}=69 \sqrt{\frac{T_{e}}{\bar{n}_{e}}}$

AIII-7

lorsque $T_{e}$ est exprimée en Kelvin, $\bar{n}_{e}$ en $\mathrm{m}^{-3}$ et $\lambda_{D}$ en $\mathrm{m}$. Dans l'ionosphère, elle va de quelques $\mathrm{mm}$ à quelques dizaines de $\mathrm{cm}$.

En utilisant des coordonnées sphériques et en remarquant que comme le problème n'a de dépendance que vis à vis de $r$, le laplacien $\nabla^{2}$ se limite à l'expression $\nabla^{2} \bullet=\frac{1}{r^{2}} \frac{d}{d r}\left(r^{2} \frac{d}{d r} \bullet\right)$, on peut récrire l'équation $A I I I-5$ sous la forme :

$\frac{1}{r^{2}} \frac{d}{d r}\left(r^{2} \frac{d \phi}{d r}\right)=\frac{\phi}{\lambda_{D}^{2}}$

AIII-8

Pour résoudre cette équation, on effectue le changement de variable $: u=\phi r$. On a alors :

$\frac{d u}{d r}=r \frac{d \phi}{d r}+\phi$ 
et :

$r^{2} \frac{d \phi}{d r}=r\left(\frac{d u}{d r}-\phi\right)$

soit :

$$
\begin{aligned}
\frac{d}{d r}\left(r^{2} \frac{d \phi}{d r}\right) & =\frac{d u}{d r}-\phi+r\left(\frac{d^{2} u}{d r^{2}}-\frac{d \phi}{d r}\right) \\
& =r \frac{d^{2} u}{d r^{2}}
\end{aligned}
$$

Par conséquent, nous pouvons écrire avec cette nouvelle variable :

$\frac{1}{r^{2}} \frac{d}{d r}\left(r^{2} \frac{d \phi}{d r}\right)=\frac{1}{r} \frac{d^{2} u}{d r^{2}}=\frac{\phi}{\lambda_{D}{ }^{2}}=\frac{u}{r \lambda_{D}{ }^{2}}$

et donc l'équation $A I I I-8$ se transforme en l'équation différentielle suivante vérifiée par $u$ :

$\frac{d^{2} u}{d r^{2}}=\frac{u}{\lambda_{D}^{2}}$

AIII-13

dont la solution générale est :

$u=A \mathrm{e}^{-\frac{r}{\lambda_{D}}}+B \mathrm{e}^{\frac{r}{\lambda_{D}}}$

Comme le potentiel décroît vers $O$ lorsque $r$ tend vers l'infini (absence de charges à l'infini), on doit avoir $B=0$, et la solution générale en $\phi$ s'écrit donc :

$\phi=\frac{A}{r} \mathrm{e}^{-\frac{r}{\lambda_{D}}}$

De plus, au voisinage de $r=0$, le potentiel $\phi$ est celui créé par la charge ponctuelle ionique : $\phi(r)=\frac{e}{4 \pi \varepsilon_{0} r}$. Nous en déduisons donc l'expression complète de $\phi$ :

$\phi(\mathrm{r})=\frac{e}{4 \pi \varepsilon_{o} r} \mathrm{e}^{-\frac{r}{\lambda_{D}}}$

La densité électronique prend alors la forme suivante :

$\tilde{n}_{e}(r)=\bar{n}_{e}\left\{1+\frac{e^{2}}{4 \pi \varepsilon_{\mathrm{o}} k_{b} T_{e}} \frac{\mathrm{e}^{-\frac{r}{\lambda_{D}}}}{r}\right\}$

On pose :

$r_{\mathrm{o}}=\frac{e^{2}}{4 \pi \varepsilon_{0} k_{b} T_{e}}$

que l'on appelle longueur de Landau (on a : $4 \pi \lambda_{D}{ }^{2} \bar{n}_{e} r_{\mathrm{o}}=1$ ). On trouve alors :

$\tilde{n}_{e}(r)=\bar{n}_{e}\left\{1+\frac{r_{\mathrm{o}}}{r} \mathrm{e}^{-\frac{r}{\lambda_{D}}}\right\}$ 
$r_{\mathrm{o}}$ correspond à la distance pour laquelle l'énergie interne $k_{b} T_{e}$ est égale à l'interaction coulombienne entre deux électrons dans le milieu, soit : $\frac{e^{2}}{4 \pi \varepsilon_{\mathrm{o}} r_{\mathrm{o}}}$. C'est la longueur critique d'interaction binaire ; cette distance apparaît comme la distance minimale d'approche entre deux électrons.

Pour $T_{e}=10^{4} \mathrm{~K}$, on trouve $r_{\mathrm{o}} \gg 10^{-6} \mathrm{~m}$, qui est très inférieur à $\lambda_{D}$ (de l'ordre $\mathrm{du} \mathrm{cm}$ ) en général. On se place dans le cas où $r_{o}<r$. Dès que $r>>\lambda_{D}$, le second terme de $\tilde{n}_{e}(r)$ devient négligeable devant 1 et l'effet du potentiel disparaît. Il y a eu effet de coupure (ou d'écrantage). Nous allons montrer que cet écrantage correspond à une neutralisation effective à grande distance de la charge de l'ion.

Si on calcule la charge électronique $Q_{e}$ répartie autour l'ion, on trouve :

$$
\begin{aligned}
Q_{e}=\int_{0}^{\infty}\left(\bar{n}_{e}-\tilde{n}_{e}(r)\right) e 4 \pi r^{2} d r & =-e \int_{0}^{\infty} \bar{n}_{e} \frac{r_{\mathrm{o}}}{r} \mathrm{e}^{-\frac{r}{\lambda_{D}}} 4 \pi r^{2} d r \\
& =-4 \pi \lambda_{D}^{2} r_{\mathrm{o}} \bar{n}_{e} e \int_{0}^{\infty} u \mathrm{e}^{-u} d u
\end{aligned}
$$

ce qui nous donne :

$Q_{e}=-e$

AIII-21

On constate que les électrons s'organisent autour de l'ion de manière à absorber le déséquilibre de charge provoqué par la présence de l'ion. A une échelle de distance supérieure à la longueur de Debye, le plasma apparaît donc comme un milieu neutre.

La longueur de Debye est la distance caractéristique qui sépare les effets individuels des effets collectifs. En effet, à une distance inférieure à $\lambda_{D}$, nous nous trouvons dans la sphère de Debye et le comportement des électrons est contraint par les interactions binaires avec les particules chargées dans cette sphère. A une distance supérieure par contre, l'écrantage a pour effet d'annuler les interactions et les particules au sein de la sphère de Debye sont vues comme des particules ayant le même comportement. De fait, on peut voir le plasma comme un milieu constitué de "pseudo-particules" de diamètre $\lambda_{D}$ sans interactions ; le comportement collectif correspond alors au mouvement de ces "pseudo-particules".

Le nombre $N$ d'électrons dans la sphère de Debye est :

$N=\frac{4}{3} \pi \lambda_{D}^{3}=\frac{1}{3} \frac{\lambda_{D}}{r_{o}}$

Dans les plasmas planétaires, nous avons $r_{o}<<\lambda_{D}$ et donc nous avons un très grand nombre d'électrons dans la sphère de Debye. On définit alors le paramètre plasma: $\Lambda=\frac{\lambda_{D}}{r_{o}}$ qui caractérise ce nombre d'électrons. Les plasmas auxquels nous pouvons appliquer les développements faits dans ce chapitre correspondent alors aux plasmas pour lesquels nous avons : $\Lambda>>1$. 
Si on veut étudier un tel plasma, il faut donc sonder le milieu avec une résolution plus faible que celle des "pseudo-particules" et donc utiliser des longueurs d'onde supérieures à $\lambda_{D}$, sinon on n'étudiera que le mouvement individuel des ions et des électrons.

\section{FRÉQUENCE PLASMA}

La mise en présence de charges de signes opposés entraîne des déplacements de charges sous l'effet de forces attractives, et donc est une source d'oscillations dans le plasma. Ces oscillations de plasma, qui sont induites par des effets à longue distance des potentiels coulombiens, sont la caractéristique de ces phénomènes collectifs. Supposons que les ions restent fixes. La séparation de charge locale crée un champ électrique de rappel, qui agit essentiellement sur les électrons, du fait de la faible mobilité des ions et de la grande variabilité temporelle de ce champ.

Nous considérerons que la densité ionique reste uniforme, égale à $\bar{n}_{i}$, et que la densité électronique subit une perturbation par rapport à la densité uniforme $\bar{n}_{e}$ donnée par : $\tilde{n}_{e}(r)=\bar{n}_{e}+\delta n_{e}(r)$, avec comme contrainte que la neutralité du plasma à l'équilibre entraine l'égalité $\bar{n}_{e}=\bar{n}_{i}$.

La perturbation de densité crée un champ électrique dont l'expression est obtenue à partir du théorème de Gauss :

$\vec{\nabla} \cdot \vec{E}=\frac{\left(\bar{n}_{i}-\tilde{n}_{e}\right) e}{\varepsilon_{\mathrm{o}}}=-\frac{e}{\varepsilon_{\mathrm{o}}} \delta n_{e}(r)$

AIII-23

Ce champ est du premier ordre en la perturbation $\delta n_{e}$ et il agit en contre-réaction sur les électrons pour les mettre en mouvement. La vitesse de déplacement des électrons $\overrightarrow{v_{e}}$ qui est induite par ce champ est alors du premier ordre en $\delta n_{e}$ et par conséquent, le terme quadratique en $\overrightarrow{v_{e}}$ dans l'équation de transport de la quantité de mouvement des électrons peut être négligé dans un développement de cette équation au premier ordre. Si de plus, on suppose dans un premier temps que le plasma est froid (effets thermiques négligeables), cette équation se réduit à la forme suivante :

$m_{e} \frac{\partial \overrightarrow{v_{e}}}{\partial t}=-e \vec{E}$

AIII-24

Par ailleurs, la quantité de matière présente dans le gaz n'est pas modifiée par ce champ, et l'équation de conservation de la matière appliquée aux électrons, entraîne :

$\frac{\partial \tilde{n}_{e}}{\partial t}+\vec{\nabla} \cdot\left(\tilde{n}_{e} \overrightarrow{v_{e}}\right)=0$

En se limitant au premier ordre en $\delta n_{e}$, cette équation devient :

$\frac{\partial \tilde{n}_{e}}{\partial t}+\bar{n}_{e} \vec{\nabla} \cdot \overrightarrow{v_{e}}=0$ 
En prenant la dérivée temporelle de l'équation AIII-26, et en intervertissant les dérivées temporelles et spatiales, on peut écrire :

$\frac{\partial^{2} \delta n_{e}}{\partial t^{2}}+\bar{n}_{e} \vec{\nabla} \cdot\left(\frac{\partial \overrightarrow{v_{e}}}{\partial t}\right)=0$

D'autre part, en utilisant l'expression de $\overrightarrow{v_{e}}$ donnée par l'équation AIII-24, l'équation AIII-27 prend la forme suivante :

$\frac{\partial^{2} \delta n_{e}}{\partial t^{2}}-\frac{e \bar{n}_{e}}{m_{e}} \vec{\nabla} \cdot \vec{E}=0$

AIII-28

En utilisant l'expression de $\vec{\nabla} \cdot \vec{E}$ donnée l'équation $A I I I-23$, nous pouvons récrire $A I I I-28$ :

$\frac{\partial^{2} \delta n_{e}}{\partial t^{2}}+\frac{e^{2} \bar{n}_{e}}{m_{e} \varepsilon_{\mathrm{o}}} \delta n_{e}=0$

On introduit la pulsation plasma :

$\omega_{p}=\sqrt{\frac{e^{2} \bar{n}_{e}}{m_{e} \varepsilon_{\mathrm{o}}}}$

et la fréquence plasma :

$f_{p}=\frac{1}{2 \pi} \sqrt{\frac{e^{2} \bar{n}_{e}}{m_{e} \varepsilon_{\mathrm{o}}}}$

AIII-31

On trouve alors que l'équation $A I I I-29$ est celle d'un oscillateur harmonique $: \ddot{x}+\omega_{p}{ }^{2} x=0$ à la fréquence plasma. Une expression approchée de la fréquence plasma, pour $\bar{n}_{e}$ exprimé en $\mathrm{m}^{-3}$ et $f_{p}$ en $\mathrm{Hz}$, est la suivante :

$f_{p}=9,1 \sqrt{\bar{n}_{e}}$

AIII-32

La séparation de charges locales entraîne donc une oscillation du plasma à la fréquence plasma $f_{p}$. Remarquons que cette fréquence ne dépend pas de $k$, c'est-à-dire du vecteur d'onde. Cela signifie que la vitesse de groupe est nulle, et que la perturbation ne peut pas se propager. Toutefois l'agitation thermique, qui a été négligée dans ce calcul, est une cause de dispersion et de propagation de cette onde.

Si nous rajoutons l'effet du gradient de pression, l'équation $A I I I-24$ devient :

$m_{e} \frac{\partial \overrightarrow{v_{e}}}{\partial t}=-e \vec{E}-\frac{1}{\tilde{n}_{e}} \vec{\nabla}\left(p_{e}\right)$

AIII-33

où $p_{e}=\tilde{n}_{e} k_{b} T_{e}$ est la pression du gaz électronique. Aux échelles de temps où nous étudions le phénomène oscillatoire, les populations n'ont pas le temps d'échanger de l'énergie et on peut considérer que le gaz électronique a un comportement adiabatique traduite par l'équation d'état suivante :

$\frac{p_{e}}{\tilde{n}_{e}^{\gamma}}=$ constante

AIII-34 
en introduisant le rapport des chaleurs spécifiques $\gamma$. Si $N_{d d l}$ est le nombre de degrés de liberté, $\gamma$ est défini par le rapport $\frac{N_{d d l}+2}{N_{d d l}}$. L'équation AIII-34 s'écrit encore :

$\frac{1}{\tilde{n}_{e}} \vec{\nabla}\left(p_{e}\right)=\gamma \frac{k_{b} T_{e}}{\tilde{n}_{e}} \vec{\nabla}\left(\tilde{n}_{e}\right)$

En nous limitant à un développement à l'ordre 1, l'équation AIII-35 devient :

$\frac{1}{\tilde{n}_{e}} \vec{\nabla}\left(p_{e}\right)=\gamma \frac{k_{b} T_{e}}{\bar{n}_{e}} \vec{\nabla}\left(\delta n_{e}\right)$

Nous pouvons alors introduire la contribution du gradient de pression dans l'équation AIII-28, qui devient :

$\frac{\partial^{2} \delta n_{e}}{\partial t^{2}}-\frac{e \bar{n}_{e}}{m_{e}} \vec{\nabla} \cdot \vec{E}-\gamma \frac{k_{b} T_{e}}{m_{e}} \vec{\nabla} \cdot\left\{\vec{\nabla}\left(\delta n_{e}\right)\right\}=0$

soit après simplification :

$\frac{\partial^{2} \delta n_{e}}{\partial t^{2}}-\gamma \frac{k_{b} T_{e}}{m_{e}} \nabla^{2}\left(\delta n_{e}\right)+\frac{e^{2} \bar{n}_{e}}{m_{e} \varepsilon_{\mathrm{o}}} \delta n_{e}=0$

AIII-38

Nous trouvons donc une équation légèrement différente de l'équation AIII-29 où un terme susceptible de provoquer la propagation de l'onde apparaît. En effet, si nous cherchons la solution sous la forme :

$\delta n_{e}=\alpha \mathrm{e}^{i(\vec{k} \vec{r}-\omega t)}$

AIII-39

l'équation AIII-38 nous fournit la relation de dispersion suivante :

$\omega^{2}=\omega_{p}^{2}+\gamma \frac{k_{b} T_{e}}{m_{e}} k^{2}=\omega_{p}^{2}\left(1+\gamma \lambda_{D}^{2} k^{2}\right)$

AIII-40

$c_{e}=\sqrt{\gamma \frac{k_{b} T_{e}}{m_{e}}}$ est la vitesse du son dans la gaz électronique, et correspond à la vitesse à laquelle l'information thermique se propage au sein de ce gaz. L'équation AIII-38 (et son interprétation en terme de relation de dispersion AIII-40) traduit donc le fait que l'oscillation est produite localement et qu'elle est ensuite propagée au sein du gaz électronique par les fluctuations thermiques. L'expression de la vitesse de groupe $v_{g}$ de l'onde vaut :

$v_{g}=\frac{d \omega}{d k}=\gamma \frac{k_{b} T_{e}}{m_{e}} \frac{k}{\omega}=c_{e}^{2} \frac{k}{\omega}=\frac{c_{e}^{2}}{v_{\phi}}$

AIII-41

où $v_{\phi}=\frac{k}{\omega}$ représente la vitesse de phase de cette onde. La relation de dispersion définit donc pour un vecteur d'onde donné une fréquence que l'on appelle la fréquence électronique et qui se traduit dans le domaine spectral par une raie appelée raie de plasma (voir figure AIII-2). 
Dans le cas où un champ magnétique contrôle le mouvement des particules, la relation de dispersion $A I I I-40$ est modifiée et devient :

$\omega^{2}=\omega_{p}^{2}+\omega_{c}^{2} \sin \theta+\gamma \frac{k_{b} T_{e}}{m_{e}} k^{2}$

où $\omega_{c}$ est la gyrofréquence électronique et $\theta$ est l'angle entre la ligne de champ magnétique et le vecteur d'onde. L'utilisation que l'on peut faire de cette relation de dispersion sera présentée dans le paragraphe consacré au sondage électromagnétique.

\section{ONDE IONIQUE}

Jusqu'à présent, nous avons considéré que les ions restent immobiles du fait de leur masse importante comparée à celle des électrons. Nous avons pu alors étudier le comportement des électrons induit par les fluctuations de charge et en particulier nous avons "balayé" la partie haute du spectre des fréquences auxquelles le plasma est sensible. La masse des ions joue comme un filtre passe-bas qui les rend insensible à cette partie du spectre, mais les fluctuations de charges sont également à l'origine d'oscillations basse fréquence auxquelles les ions répondent. Par contre, les électrons sont insensibles à cette partie basse du spectre, car leur masse joue comme un filtre passe-haut. Nous allons donc considérer que les électrons, qui oscillent à une fréquence proche de la fréquence plasma, sont en moyenne, à une échelle de temps grande devant celle de l'oscillation plasma que nous préciseront ultérieurement, immobile et à l'équilibre thermodynamique tel que donné par AIII-2 autour d'un ion qui est en mouvement sous l'effet de la perturbation induite par les fluctuations de charges.

Cette fois-ci nous prendrons : $\tilde{n}_{i}=\bar{n}_{i}+\delta n_{i}, \tilde{n}_{e}$ donné par AIII-3 (car l'hypothèse sous-jacente est toujours vérifiée), avec comme contrainte que la neutralité du plasma à l'équilibre entraîne l'égalité $\bar{n}_{i}=\bar{n}_{e}$. Nous pouvons alors récrire l'équation de Poisson AIII-5 sous la forme :

$\nabla^{2} \phi=-e \frac{\tilde{n}_{i}-\tilde{n}_{e}}{\varepsilon_{\mathrm{o}}}=\frac{\bar{n}_{\mathrm{e}} e^{2}}{\varepsilon_{\mathrm{o}} k_{b} T_{e}} \phi(r)-\frac{e}{\varepsilon_{o}} \delta n_{i}$

AIII-43

ou :

$\nabla^{2} \phi=\frac{\phi}{\lambda_{D}^{2}}-\frac{e}{\varepsilon_{o}} \delta n_{i}$

AIII-44

En s'inspirant des écritures pour l'électron, on peut écrire l'équation de conservation de l'ion sous la forme :

$\frac{\delta n_{i}}{\partial t}+\bar{n}_{e} \vec{\nabla} \cdot \overrightarrow{v_{i}}=0$

AIII-45

où $\overrightarrow{v_{i}}$ est la vitesse de l'ion. Par ailleurs, en s'inspirant des développement pour l'électron, on peut écrire l'équation de transport de la quantité de mouvement de l'ion :

$m_{i} \frac{\partial \overrightarrow{v_{i}}}{\partial t}=e \vec{E}-\gamma_{i} \frac{k_{b} T_{i}}{\bar{n}_{e}} \vec{\nabla}\left(\delta n_{i}\right)$ 
où nous avons utilisé pour l'ion l'équation d'état suivante :

$\frac{p_{i}}{\tilde{n}_{i}^{\gamma_{i}}}=$ constante

$\gamma_{i}$ étant le rapport des chaleurs spécifiques de l'ion. En manipulant l'équation de conservation de l'ion comme nous l'avons fait pour celle de l'électron, nous pouvons écrire :

$\frac{\partial^{2} \delta n_{e}}{\partial t^{2}}+\frac{e \bar{n}_{e}}{m_{i}} \vec{\nabla} \cdot \vec{E}-\gamma_{i} \frac{k_{b} T_{i}}{m_{i}} \vec{\nabla} \cdot\left\{\vec{\nabla}\left(\delta n_{i}\right)\right\}=0$ AIII-48

et nous aboutissons alors au système différentiel suivant :

$$
\left\{\begin{array}{l}
\frac{\partial^{2} \delta n_{i}}{\partial t^{2}}-\gamma_{i} \frac{k_{b} T_{i}}{m_{i}} \nabla^{2}\left(\delta n_{i}\right)-\frac{e \bar{n}_{e}}{m_{i}} \nabla^{2} \phi=0 \\
\nabla^{2} \phi=\frac{\phi}{\lambda_{D}^{2}}-\frac{e}{\varepsilon_{o}} \delta n_{i}
\end{array}\right.
$$

Comme précédemment, nous cherchons une famille de solution de la forme :

$$
\left\{\begin{array}{l}
\hat{\delta} n_{i}=\alpha \mathrm{e}^{i(\vec{k} \vec{r}-\omega t)} \\
\hat{\phi}=\beta \mathrm{e}^{i(\vec{k} \vec{r}-\omega t)}
\end{array}\right.
$$

En introduisant ces solution dans le système $A I I I-49$, nous trouvons :

$$
\left\{\begin{array}{l}
-\omega^{2} \hat{\delta} n_{i}+\gamma_{i} \frac{k_{b} T_{i}}{m_{i}} k^{2} \hat{\delta n_{i}}+\frac{e \bar{n}_{e}}{m_{i}} k^{2} \hat{\phi}=0 \\
k^{2} \hat{\phi}=-\frac{\hat{\phi}}{\lambda_{D}^{2}}+\frac{e}{\varepsilon_{o}} \hat{\delta n_{i}}
\end{array}\right.
$$

Ce qui nous donne :

$$
\hat{\phi}=\frac{e}{\varepsilon_{o}} \frac{\lambda_{D}{ }^{2}}{1+\lambda_{D}{ }^{2} k^{2}} \hat{\delta} n_{i}
$$

et la relation de dispersion suivante :

$$
\omega^{2}=\gamma_{i} \frac{k_{b} T_{i}}{m_{i}} k^{2}+\frac{e^{2} \bar{n}_{e} \lambda_{D}^{2}}{\varepsilon_{o} m_{i}} \frac{k^{2}}{1+\lambda_{D}^{2} k^{2}}
$$

soit, d'après la définition de la longueur de Debye :

$$
\omega^{2}=k^{2} \frac{\gamma_{i} k_{b} T_{i}+\frac{k_{b} T_{e}}{1+\lambda_{D}^{2} k^{2}}}{m_{i}}
$$


En comparant à l'équation de dispersion obtenue dans la cadre de la fréquence plasma, on trouve que l'onde qui se développe est une onde à basse fréquence. En effet, la vitesse de phase de cette onde est de l'ordre de grandeur de $c_{i}=\sqrt{\gamma_{i} \frac{k_{b} T_{i}}{m_{i}}}$, la vitesse du son dans le gaz ionique. L'équation AIII-54 traduit le fait que l'énergie thermique des électrons est suffisante pour entretenir une oscillation ionique dans l'ionosphère. Le principe est que le nuage électronique oscille à grande fréquence, entraînant les ions à une fréquence acoustique plus faible. C'est pourquoi la pulsation dépend à la fois de la température électronique et de la masse ionique. Le mouvement des ions se fait vers les zones de plus grande concentration locale d'électrons, si bien qu'il existe localement des compressions d'ions, et un champ de pression, dont la contribution à l'onde acoustique ionique est le terme en $T_{i} / m_{i}$. En supposant que les ions et les électrons ont la même température, le rapport des vitesses du son $\frac{c_{e}}{c_{i}}$ est de l'ordre de grandeur de la racine du rapport des masses $\sqrt{\frac{m_{i}}{m_{e}}}$. Or dans les deux ondes étudiées, nous avons des vitesses de phases de l'ordre de grandeur des vitesses du son. Par conséquent, pour un vecteur d'onde donné $\vec{k}$, nous pouvons écrire :

$\frac{\omega_{i}}{\omega_{e}}=\frac{\frac{\omega_{i}}{k}}{\frac{\omega_{e}}{k}}=\frac{v_{\phi i}}{v_{\phi e}} \propto \frac{c_{e}}{c_{i}} \propto \sqrt{\frac{m_{e}}{m_{i}}} \cong 40$

AIII-55

où $\omega_{e}$ représente la pulsation de l'onde plasma et $\omega_{i}$ celle de l'onde ionique. Dans l'hypothèse où on a un vecteur d'onde correspondant à une longueur d'onde très grande devant $\lambda_{D}$, l'équation $A I I I-54$ se simplifie et devient :

$\omega^{2}=k^{2} \frac{\gamma_{i} k_{b} T_{i}+k_{b} T_{e}}{m_{i}}$

AIII-56

Dans ce cas, la vitesse de groupe est constante, indépendante du vecteur d'onde ; elle est de l'ordre de grandeur de la vitesse du son dans le gaz ionique.

\section{SONDAGES ÉLECTROMAGNÉTIQUES AU SOL}

\section{IONOSONDE}

Supposez qu'on vous demande de sonder l'ionosphère avec une onde électromagnétique. Comment choisir la longueur d'onde ? La limite basse est la fréquence de plasma. En dessous de cette fréquence, de l'ordre de quelques $\mathrm{MHz}$, l'onde ricochera sur l'ionosphère, phénomène utilisé avant la généralisation des satellites pour des communications à distance. Dans la gamme du MHz, l'onde sera réfléchie à l'altitude à laquelle elle est égale à la fréquence de plasma (fréquence proportionnelle à $\sqrt{n_{e}}$ ). Ainsi, nous pouvons faire des 
sondages simples donnant le profil de densité électronique (puisqu'on peut mesurer le temps d'aller retour de l'onde, on connaît l'altitude de réflexion). L'instrument qui fait cette mesure n'est pas appelé radar, mais ionosonde. Il permet de sonder l'ionosphère tant que la densité électronique croît. Pour cela, on envoie une onde vers l'ionosphère, dont on augmente la fréquence. La figure AIII-1 montre le résultat d'un tel sondage radio, le 18 juin 1997 à 12:40 TU. L'ordonnée montre une hauteur virtuelle. C'est que la distance de la cible sur laquelle l'onde ricoche à l'émetteur est simplement calculée en divisant le temps de propagation par la vitesse de la lumière. L'angle de visée fait que cette distance ne correspond pas nécessairement à l'altitude. On distingue également deux échos. Ceux-ci sont dus à deux modes différents de propagation de l'onde électromagnétique dans l'atmosphère.

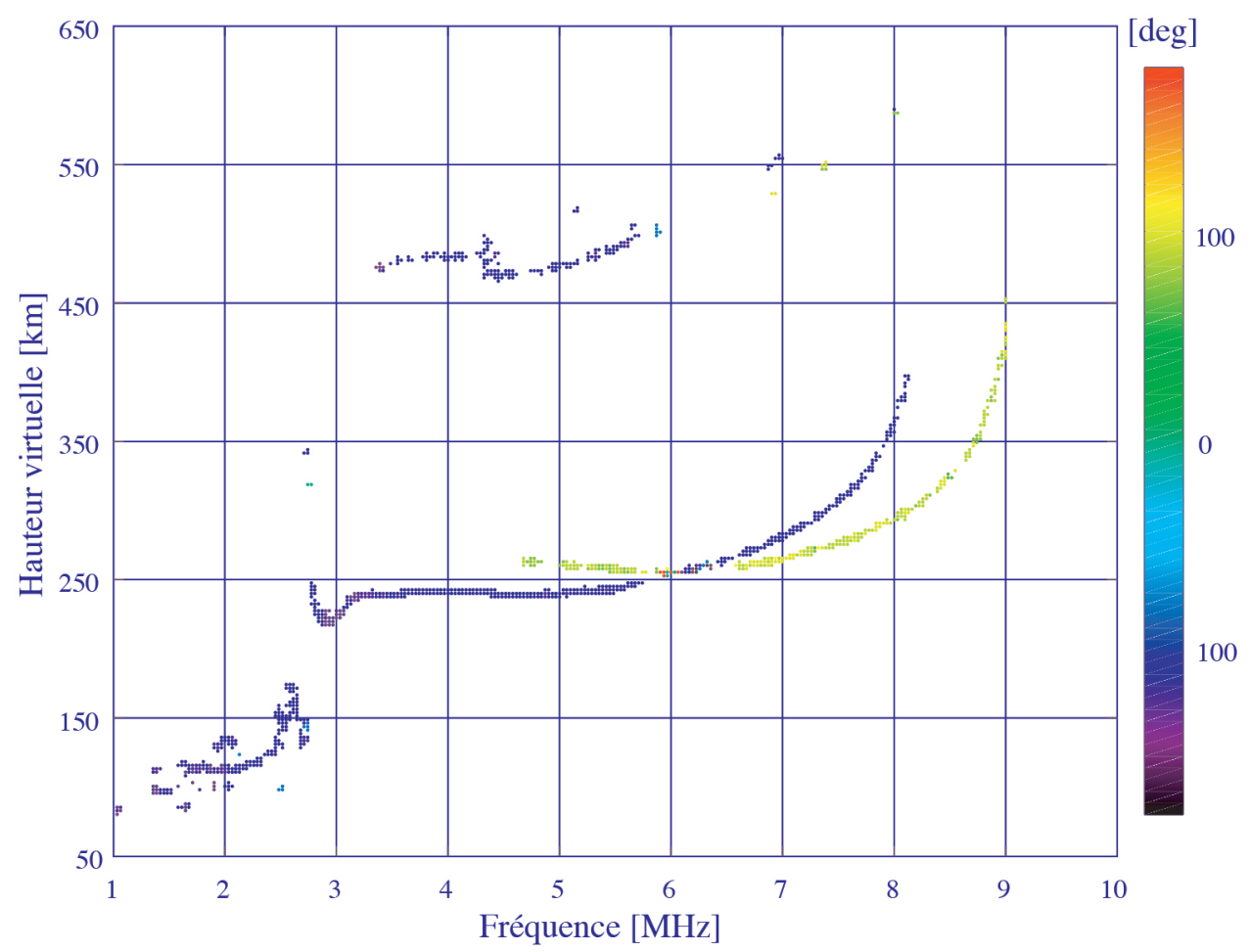

Figure AIII-1 : exploration ionosphérique par ionosonde

(crédit : M. Rietveld, EISCAT scientific association)

Sur cette figure, on voit se former une région $E$ à basse altitude. L'onde ne peut bien sûr atteindre le minimum entre la région $E$ et la région $F$, et ricoche, vers $200 \mathrm{~km}$, là où la densité électronique devient de nouveaux supérieure à celle de la région $E$. En utilisant la formule $A I I I-31$, on peut facilement remonter à une bonne estimation de la densité électronique. 


\section{LA DIFFUSION INCOHÉRENTE}

Au pic de densité, la fréquence de plasma est de l'ordre de quelques $\mathrm{MHz}$, et les ondes ne peuvent plus être réfléchies. Comment sonder l'ionosphère au dessus de ce pic, et ce de façon permanente en un même lieu ? Comment accéder à davantage de caractéristiques de l'ionosphère que la seule densité électronique ? Au moyen de la diffusion Thomson, appelée aussi diffusion incohérente.

\section{a. Principe de la diffusion incohérente}

Si on envoie dans l'ionosphère une onde électromagnétique d'une fréquence supérieure à la fréquence de plasma au pic de densité électronique, l'onde traverse l'ionosphère. Sur son passage, elle excite les électrons (et les ions, mais ceux ci sont très massifs), qui se mettent à osciller, en diffusant de l'énergie. Cette énergie diffusée pourrait être détruite par l'onde diffusée par un autre électron en opposition de phase avec le premier, mais la distribution des électrons étant aléatoire, il y a peu de chance que cela arrive : l'onde est donc diffusée par les inhomogénéités de l'ionosphère. La distribution des électrons étant aléatoire, cette diffusion a été appelée diffusion incohérente.

Quelle est la fréquence maximale possible? L'onde électromagnétique doit pouvoir exciter le plasma, mais si sa longueur d'onde est inférieure à la longueur de Debye, elle verra des électrons et des ions indépendants. Ces particules émettront à la fréquence reçue, sans donner d'information sur le milieu. Le spectre réémis par le plasma ainsi excité sera proche d'une gaussienne, correspondant simplement à l'effet Doppler lié à la distribution des vitesses ! Le choix est donc nécessairement celui d'une longueur d'onde plus grande que la longueur de Debye, qui verra les "pseudo-particules" caractéristiques du plasma. Les électrons excités réémettront l'énergie autour de la fréquence d'émission, mais avec des caractéristiques fonction du plasma, en particulier un élargissement acoustique-ionique (l'oscillation de l'ion est communiquée aussi aux électrons et de l'extérieur, la "pseudo particule" vibre avec cette pulsation acoustique), et une émission autour de la raie de plasma (caractéristique de la densité et de la température des électrons). Prenons un un plasma de concentration $n_{e}=10^{12} \mathrm{~m}^{-3}$ et de température $T_{e}=1600 \mathrm{~K}$. On trouve alors $\lambda_{D}=0,27 \mathrm{~cm}$ et $v_{D}=\frac{c}{\lambda_{D}}=10^{11} \mathrm{~Hz}$. Il faut donc travailler avec une fréquence inférieure à $10^{11} \mathrm{~Hz}$, soit par exemple $1 \mathrm{GHz}$.

\section{b. Quelles informations tirer de la diffusion incohérente?}

La forme générale du spectre est représentée sur la partie droite de la figure AIII-2. Le spectre ionique-acoustique est centré autour de la fréquence d'émission, d'une largeur de quelques kilohertz. De chaque côté, à environ huit $\mathrm{MHz}$, apparaît la raie de plasma. Jusqu'à présent, les études sur la raie de plasma n'ont pas abouti à des mesures de routine : cette raie est fine et en mouvement permanent, ce qui rend sa détection difficile.

Mais toute déformation du spectre ionique est porteuse d'information : sa surface totale est fonction du nombre de "réémetteurs", c'est à dire de la densité électronique ; son 
élargissement peut être dû à des températures ioniques élevées ; comme le nombre de collisions est aussi fonction de la masse des ions, cette largeur donne des informations sur $m_{i}$ et donc sur la composition ionique. Un déséquilibre thermique entre ions et électrons augmente la taille verticale des pics de chaque côté du spectre, et puisque $T_{i}$ est connu par la largeur du spectre, on en déduit $T_{e}$. Un effet Doppler - c'est à dire une vitesse d'ensemble des ions le long de la ligne de visée - décalera en fréquence le spectre sans en altérer la forme. Les informations de base obtenues à partir de ce spectre sont donc $n_{e}, T_{e}, T_{i}, v_{i}$ et la composition ionique. Les incertitudes sont de l'ordre de $1 \%$ sur $n_{e}, 1$ à $10 \mathrm{~m} \mathrm{~s}^{-1}$ sur $v_{i}$, une dizaine de degrés sur les températures, quelques pour cents sur la composition ionique.
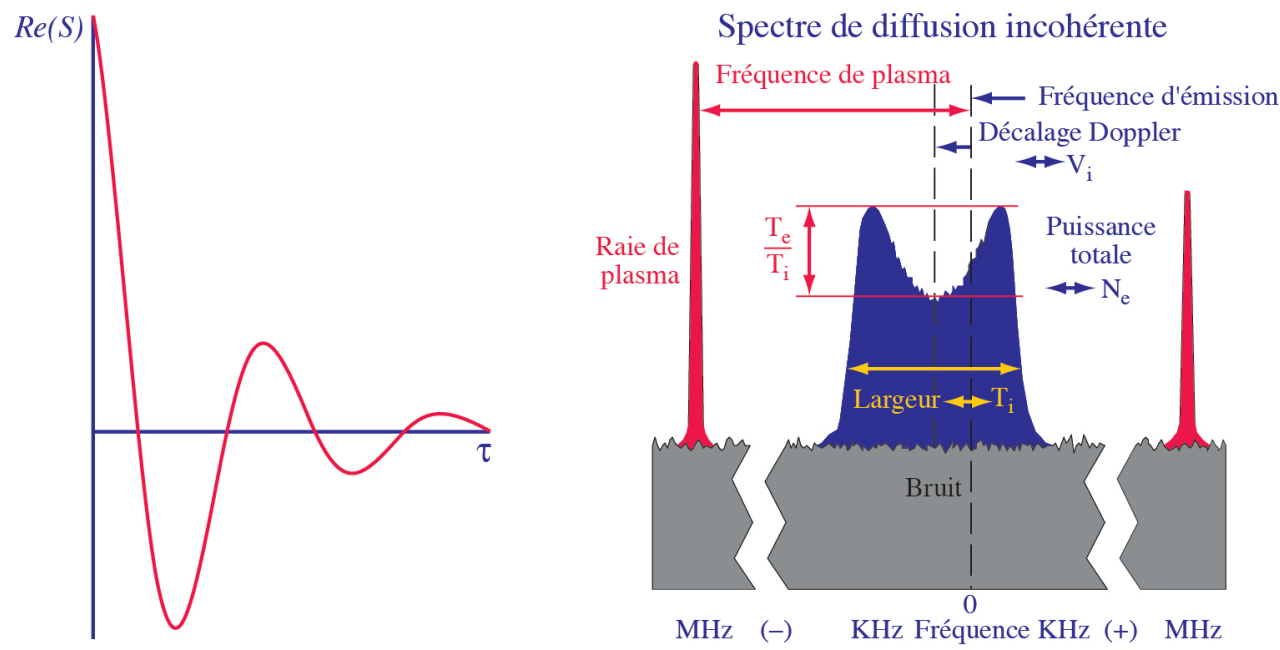

Figure AIII-2 : à droite, on a représenté le spectre de diffusion incohérent avec les informations auxquelles il permet d'accéder et on a tracé à gauche, la forme de sa transformée de Fourier qui est la fonction d'autocorrélation du signal rétrodiffusé par le plasma.

L'analyse standard des données se fait en cherchant les paramètres physiques qui ajustent au mieux une fonction d'autocorrélation théorique à la fonction mesurée, au sens des moindre carrés.

$\mathrm{Au}$ dessous de $120 \mathrm{~km}$, les températures ioniques et électroniques s'égalisent. Dans ce cas, on déduit un paramètre supplémentaire, la fréquence de collisions ions-neutres en région $E$. Nous avons présenté au chapitre $V I$ (figure $V I-18$ ) les résultats d'un sondage de l'ionosphère avec le radar UHF, fonctionnant à $931 \mathrm{MHz}(\lambda=30 \mathrm{~cm})$ et le radar VHF, fonctionnant à $224 \mathrm{MHz}(\lambda=1,3 \mathrm{~m})$ de l'association EISCAT. Sur cette figure, nous avons tracé en fonction du temps et de l'altitude, les valeurs des principaux paramètres du plasma, estimées à partir des fonctions d'autocorrélation selon la méthode décrite sur la figure AIII-2.

De ces paramètres issus directement de la mesure, on tire beaucoup d'autres enseignements au moyen des équations vues dans les chapitres précédents: composition ionique, convection, dynamique, ... 


\section{c. Mise en ouvre}

La mise en œuvre de la diffusion incohérente se révèle un exercice difficile et coûteux. Mais les informations qu'on en tire sont tellement nombreuses qu'il existe au plan international une grande motivation.

La première réalisation a été celle du National Bureau of Standards, installé à Jicamarca, au Pérou, pour être proche de l'équateur magnétique, et sonder ainsi perpendiculairement au champ. Cet instrument fonctionne depuis 1962, avec une antenne composée de 18000 dipôles couvrant une surface de 300x300 m. Puis, l'année suivante, le Lincoln Laboratory of Massachusetts ouvrait son radar (Millestone Hill), avec une antenne de $70 \mathrm{~m}$ de diamètre. En 1964, l'université Cornell installait à Porto Rico ce qui reste la plus grande antenne du monde, $300 \mathrm{~m}$ de diamètre. La France fut le premier pays européen à se lancer dans l'aventure, avec son sondeur de Nançay/Saint Santin, en 1965. Elle innovait, avec une antenne émettrice / réceptrice et deux antennes réceptrices, acquérant un savoir faire qui lui a permis de participer activement au projet EISCAT (European Incoherent SCATter), situé en zone aurorale, et qui possède un émetteur récepteur en Norvège, un récepteur en Suède et un en Finlande. Cette configuration unique lui permet de faire des observations vectorielles, et lui a donné un rôle de leader mondial. EISCAT est géré par la France, l'Allemagne, la Grande Bretagne, la Norvège, la Suède et la Finlande.

EISCAT étudie l'ionosphère aurorale depuis 1981 sur une bande de dix degrés de latitude, entre 70 et 600 kilomètres d'altitude. Il est composé de trois antennes : l'émetteur-récepteur, une parabole de 32 mètres de diamètre travaillant à $931 \mathrm{MHz}$ se trouve à Troms $\varnothing$, à 69,35 de latitude nord. Il délivre une puissance de $2 \mathrm{MW}$ en des impulsions de quelques dizaines de micro secondes. Deux antennes réceptrices de même dimension sont situées à Kiruna (Suède) et Sodankylä (Finlande). Cette configuration unique au monde permet d'observer un point de l'atmosphère depuis trois directions différentes, et donc d'étudier les phénomènes en trois dimensions et pas seulement le long de la ligne de visée comme les autres radars de ce type dans le monde. En plus de ce radar à Ultra Haute Fréquence (UHF), EISCAT dispose à Troms $\emptyset$ d'une antenne de 120 mètres de long en quatre pans rectangulaires qui, avec sa fréquence VHF de $224 \mathrm{MHz}$, permet de faire des mesures jusqu'à plus de 1000 kilomètres d'altitude.

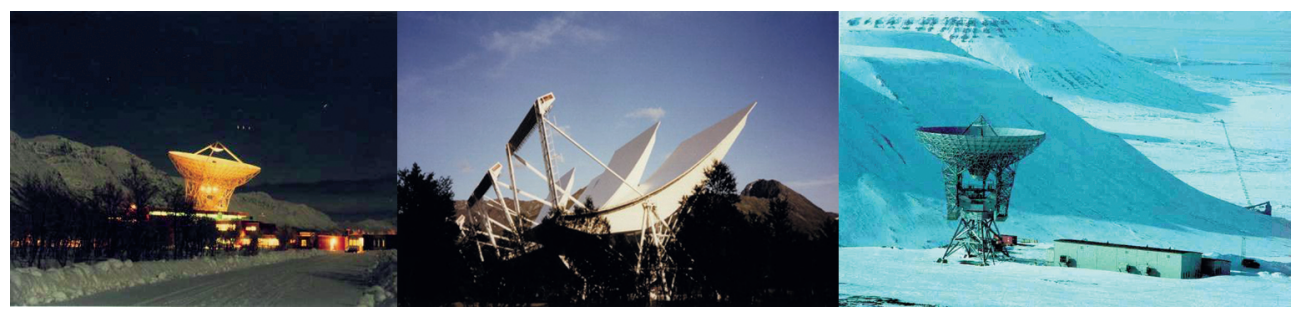

Figure AIII-3 : les antennes UHF (à gauche), VHF (au centre) de Tromsø et l'antenne ESR à Svalbard 
EISCAT a inauguré en août 1996 une antenne travaillant à $450 \mathrm{MHz}$ sur l'île de Svalbard (78,09 ${ }^{\circ}$ de latitude nord). Pour ce dernier projet, appelé Eiscat Svalbard Radar (ESR), le Japon a rejoint les six pays fondateurs. Sa position permettra de comprendre ce qui se passe dans le cornet polaire, et donc de mieux saisir le couplage entre l'ionosphère et la magnétosphère. Une seconde antenne a été construite en 1999.

\section{CHAUFFAGE ÉLECTRONIQUE}

En liaison avec un radar à diffusion incohérente, EISCAT offre la ressource unique d'une antenne de chauffage électronique de l'ionosphère. Il s'agit cette fois d'émettre une onde à la fréquence de plasma, qui va mettre en résonance des électrons. L'ionosphère est "chauffée". Le radar permet de mesurer la variation des paramètres sous l'effet du chauffage, et d'en déduire comment se fait le transfert d'énergie dans l'ionosphère.

\section{RADARS COHÉRENTS}

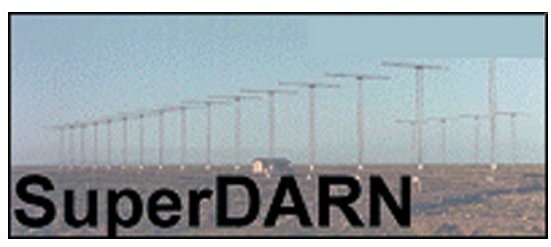

Les radars incohérents peuvent faire des mesures quel que soit l'angle entre le champ magnétique et le vecteur d'onde. Mais lorsque l'onde rencontre des inhomogénéités de plasmas dans des régions où elle se propage perpendiculairement au champ magnétique, une diffusion de Bragg se produit qui résulte en une diffusion cohérente de l'onde incidente ; c'est le principe des radars cohérents, qui détectent donc également le signal rétrodiffusé par les fluctuations d'ionisation présentes dans l'ionosphère. Ces radars fonctionnent dans la gamme $\mathrm{HF}$ ( 8 à $20 \mathrm{MHz}$ ). Le principe autorise des puissances d'émissions plus faibles que pour la diffusion incohérente $(600 \mathrm{~W}$ contre 100 à $300 \mathrm{~kW}$ en puissance moyenne), et rend la technologie plus simple de mise en oeuvre (et donc le prix moins élevé).

Cette technique a été développée à la fin des années 70 par plusieurs groupes français. Un projet international a permis la mise sur pied d'un réseau de radars cohérents, dont la configuration est montrée sur la figure A2-6, et qui possède la particularité de couvrir l'ionosphère sur plus de 12 heures de secteur angulaire en longitude à un instant donné, avec des observations conjuguées dans l'hémisphère sud. Ce réseau, appelé SuperDARN permet l'étude simultanée d'une grande zone de l'ionosphère (environ 10 heures de secteur angulaire en longitude), et donc en particulier l'étude de sa dynamique. Nous en avons montré des exemples au cours des divers chapitres de ce livre. 

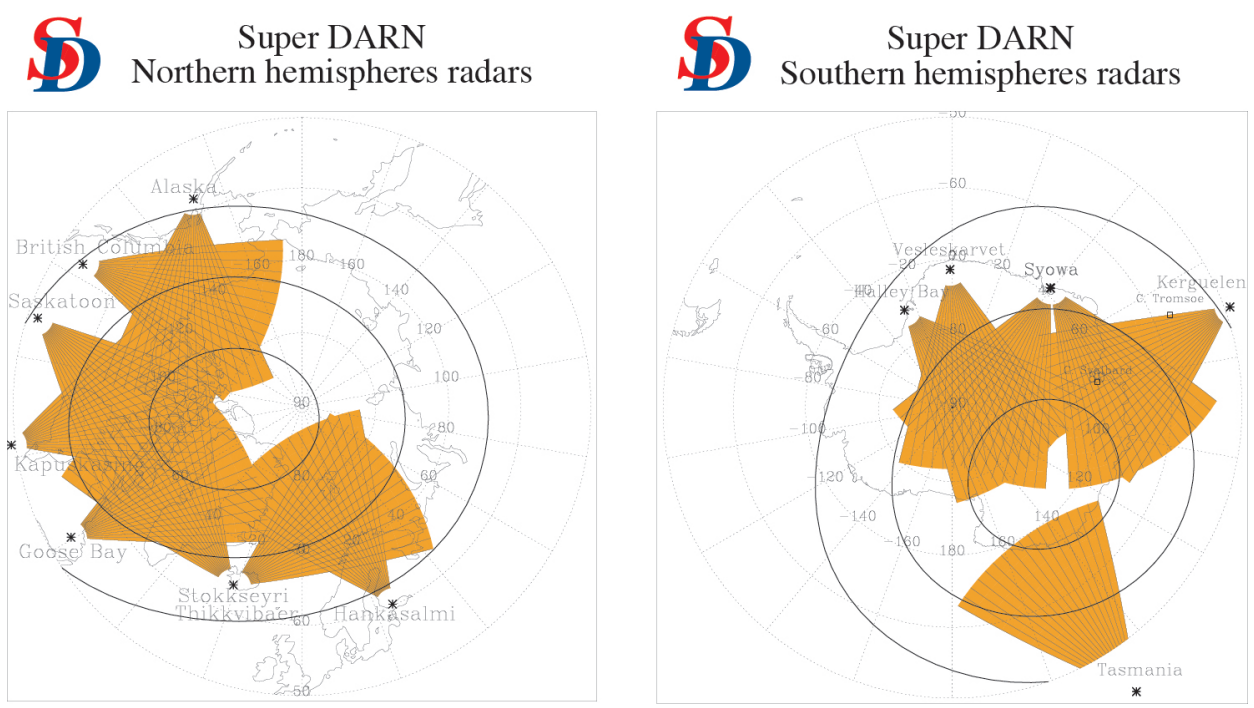

Figure AIII-4 : location des antennes du réseau SuperDARN

A gauche, dans l'hémisphère nord les latitudes et longitudes sont: Finlande $(60,20 ; 24,60)$; Islande est 64,$0 ; 33,60)$; Islande ouest $(64,43 ; 337,98)$; Goose Bay est $(53,40 ; 299,50)$; Kapuskasing $(49,41 ; 277,55)$; Saskatoon est $(51,50 ; 251,50)$; Alaska $(57,80 ; 207,60)$. A droite figure les antennes de l'hémisphère sud.

\section{BIBLIOGRAPHIE}

L'ensemble des techniques de sondage ionosphérique est décrit dans :

Radio technique for probing the terrestrial ionosphere, R.D. Hunsucker, Springer Verlag.

Concernant la technique de la diffusion incohérente, on pourra lire l'article fondateur de J.J. Thomson lui même :

Conduction of electricity through gases, Thomson J.J., London cambridge Univ. Press., 21, 1906.

L'idée d'appliquer la diffusion Thomson aux radars est due à :

Gordon, W.E., Proc. IRE, 46, 1824-1829, 1958.

Deux articles, ancien pourtant, donnent une idée très clair du principe de la diffusion incohérente :

Theory of waves incoherently scattered, Bauer P., Phil. Trans. R. Soc. Lond., 280, 167-191, 1975.

Detection and estimation proctices in radio and radar astronomy, Hagfors T., Proc. IEEE, 58, 743-759, 1970. 
La mesure de la raie de plasma s'appuie sur les considérations théoriques de :

Yngvesson, K.O. and Perkins, F.W., Radar Thomson scatter studies of photoelectrons in the ionosphere and Landau Damping, J. Geophys. Res., 73, 97-102, 1968.

Un premier bilan de l'activité scientifique autour de EISCAT a été écrit dans :

EISCAT : early history and the first ten years of operation, H. Rishbeth and A.P. Van Eycken, Journal of Atm. Terr. Phys., 55, 525-542, 1993.

La diffusion cohérente et le réseau SuperDarn sont bien décrits par :

Darn / SuperDarn: A global view of high-latitude convection, Greenwald, R.A. et al., Space Sci. Rev., 71, 763-796, 1995.

Le chauffage électronique dans :

Reitveld, M. and L. Kalliopuska (eds), 4th European Heating Seminar, Ramfjordmoen, Norway, May 16-19, 1995, Extended Abstracts, Report, 12 (1995), Department of Physics, University of Oulu, Finland, 1995.

Enfin, sur la toile, on peut se connecter à EISCAT et, de là, voyager sur tous les instruments mondiaux pour l'aéronomie :

http://www.eiscat.no.index.html 
